\title{
Chemical tapering of polymer optical fiber
}

\author{
Affa Rozana Abdul Rashid ${ }^{1, *}$, Amna Afiqah Nasution ${ }^{1}$, Aisyah Hanim Suranin ${ }^{1}$, Nur Athirah Taib ${ }^{1}$, Wan Maisarah \\ Mukhtar $^{1}$, Karsono Ahmad Dasuki ${ }^{1}$, and Abang Annuar Ehsan ${ }^{2}$ \\ ${ }^{1}$ Faculty of Science and Technology, Universiti Sains Islam Malaysia, 71800, Nilai, Negeri Sembilan, Malaysia \\ ${ }^{2}$ Institute of Microengineering and Nanoelectronics, Universiti Kebangsaan Malaysia, 43600 UKM Bangi, Selangor, Malaysia
}

\begin{abstract}
Polymer optical fibers (POFs) have significant advantages over numerous sensing applications. The key element in developing sensor is by removing the cladding of the fiber. The use of organic solvent is one of the methods to create tapered POF in order to expose the core region. In this study, the etching chemicals involved is acetone, methyl isobutyl ketone (MIBK), and acetone-methanol mixture. The POF is immersed in $100 \%, 80 \%$, and $50 \%$ of acetone and MIBK dilution. In addition, the mixture of acetone and methanol is also used for POF etching by the ratio 2:1 of the volume. Acetone has shown to be the most reactive solvent towards POF due to its fastest etching rate compared to MIBK and acetone-methanol mixture. The POF is immersed and lifted from the solution for a specific time, depending on the power loss properties for the purpose of producing unclad POF. In comparison to silica fiber optic, the advantages of POF in terms of its simple technique and easy handling enable it to produce unclad POF without damaging the core region. The surface roughness of the POF is investigated under the microscope after being immersed into different solvent. This method of chemical tapering of POF can be used as the fundamental technique for sensor development. Next, the unclad fiber is immersed into ethanol solutions in order to determine the reaction of unclad POF towards its surrounding. The findings show that this particular sensor is sensitive towards concentration changes ranging between $10 \mathrm{wt} \%$ to $50 \mathrm{wt} \%$.
\end{abstract}

\section{Introduction}

Polymer optical fibres (POFs) also known as plastic optical fibres possess many advantages over conventional silica or glass optical fibre, especially in sensing application. The wide application of POFs in sensor design tends to originate from the development of polymer technology. Their advantages which include low cost or low cost competitive system over the glass or silica optical fibre have initiated a great interest among the scientific community to further explore POF. POF is commonly found in the multi-mode fibres with large diameter, thus allowing the propagation of multiple modes $[1,2]$. The diameter of POF must be reduced by removing the cladding of the fiber to develop sensor based polymer optical fiber. The etched region of POF becomes more sensitive to the surrounding due to the evanescent wave in the cladding [3]. Etching refers to the reduction process of localised diameter, which came within any profile from linear and gradual changes to abrupt steps [4]. There are several ways to remove the cladding of POF, in which one of them is described as hot drawing method but this method does not efficiently remove the cladding because the fiber will break before any elongation occurs [4]. Hence, the best way to remove the cladding of POF is by immersing chemical etching in the organic solvent. The penetration depth of the evanescent field and the power proportion were observed to increase within the tapered region as the fiber is immersed in the testing solution. Evanescent wave in fiber optic occurs when a part is light up, whereby the incident between different refractive index of core and cladding is transmitted into the cladding rather than being reflected back which is based on the principle of total internal reflection [5]. In this particular kind of evanescent wave absorbance sensing phenomena, the measurement is calculated based on the loss of propagating light at the interface of core and cladding medium [6]. The method of etching tapers is performed using acetone, MIBK, and acetone-methanol mixture for the purpose of developing step-index POF in this project.

\section{Methodology}

The POF model adopted in this experiment is known as step-index Polyethylene Jacketed Optical. The diameter of cladding and core must be set at $1000 \mu \mathrm{m}$ and 980 $\mu \mathrm{m}$, respectively. Three types of solution have been used in the etching of polymer optical fiber, namely acetone, MIBK and acetone-methanol mixture. $4 \mathrm{~cm}$ of the jacket of the fiber is removed and placed inside a testing solution. In the first step, the POF is immersed in pure solution of acetone and MIBK. Next, the fiber is neutralized with de-ionize water. The experiment is repeated using dilute solution of $80 \%$ and $50 \%$ acetone and MIBK, respectively. On top of that, the mixture of

\footnotetext{
Corresponding author: affarozana@usim.edu.my
} 
acetone and methanol is also used in the ratio with the volume $(2: 1)$ [7] in order to study the etching effect of mixture towards the unjacketed POF. The preparation of different solutions which include ethanol should be properly done in order to study the effect of absorbing liquid on the unclad POF. In this process, the dilution of $10 \mathrm{wt} \%, 20 \mathrm{wt} \%, 30 \mathrm{wt} \%, 40 \mathrm{wt} \%$, and $50 \mathrm{wt} \%$ of each ethanol concentration are prepared.

\section{Result and Discussions}

\subsection{Effect of Acetone on Etching Rate}

Acetone is capable of removing the cladding of polymer which is composed by fluoropolymer with PMMA as its core. Fig. 1 shows the etching rate of POF that is immersed in acetone without dilution. As can be seen in the figure, it takes around $10 \mathrm{~s}$ to reach the core of the POF fiber. Next, the power loss increases abruptly as the acetone reaches and etches the core part after $10 \mathrm{~s}$. Hence, it can be concluded that it only a short time is needed to reach the core but the diameter of the fiber becomes unpredictable and fragile as a result of pure acetone. Fig. 2 and Fig. 3 show the etching rate of the fiber in acetone with concentration percentage of $80 \%$ and $50 \%$. The surrounding environment needs to be controlled which include humidity, temperature, pressure, and others. The fiber breaks and damages at a very short time because the power loss increases rapidly. The time taken to lift the fiber decreases as the concentration increases.

For $80 \%$ concentration of acetone, it takes around $1 \mathrm{~min}$ before the loss of the power output to abruptly increase. Meanwhile, the fiber does not show any abrupt change even though the fiber is immersed in the solution for 2 hours for $50 \%$ concentration of acetone as shown in Fig. 3. The graph of the power loss slightly increases from $12.13 \mathrm{~mW}$ to $12.27 \mathrm{~mW}$ with the time recorded at $85 \mathrm{~s}$ to $90 \mathrm{~s}$, respectively. However, the power output still fail to show any changes even with 2 hours of immersion.

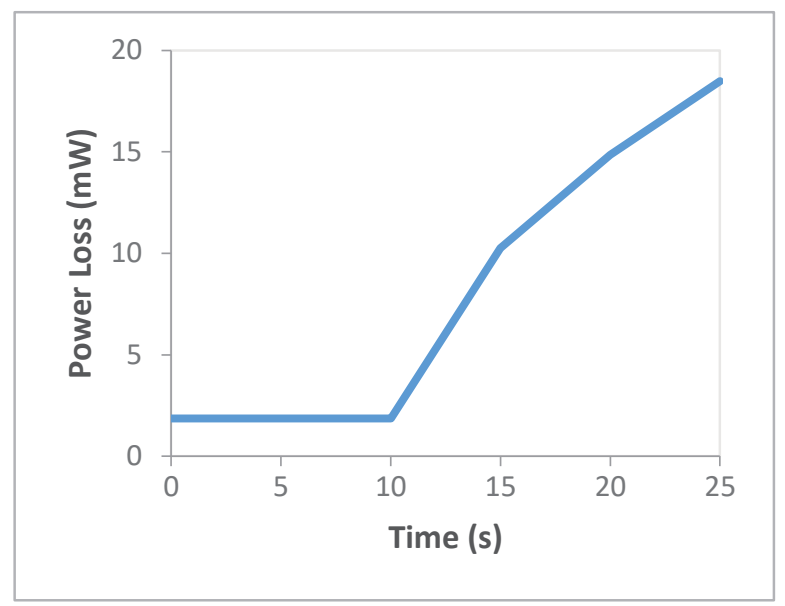

Fig. 1. Graph of power loss vs etching time of POF in $100 \%$ Acetone

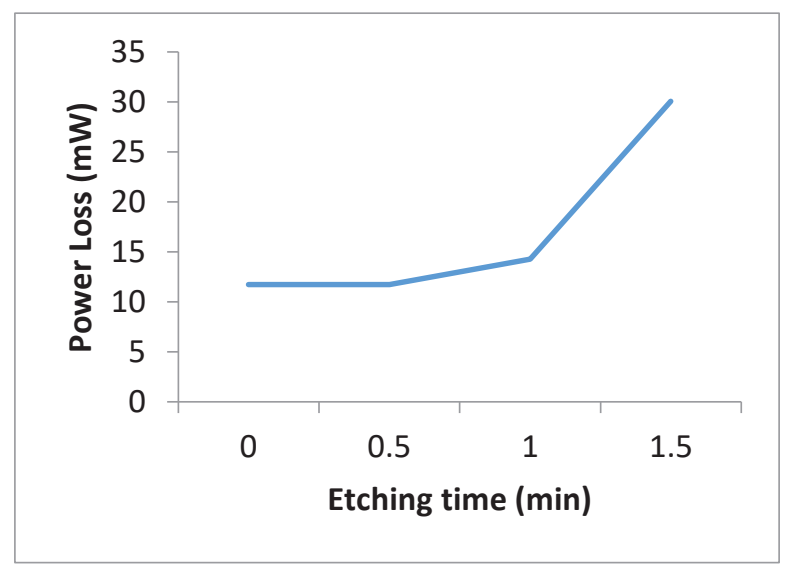

Fig. 2. Graph of power loss vs etching timeof POF in $80 \%$ Acetone

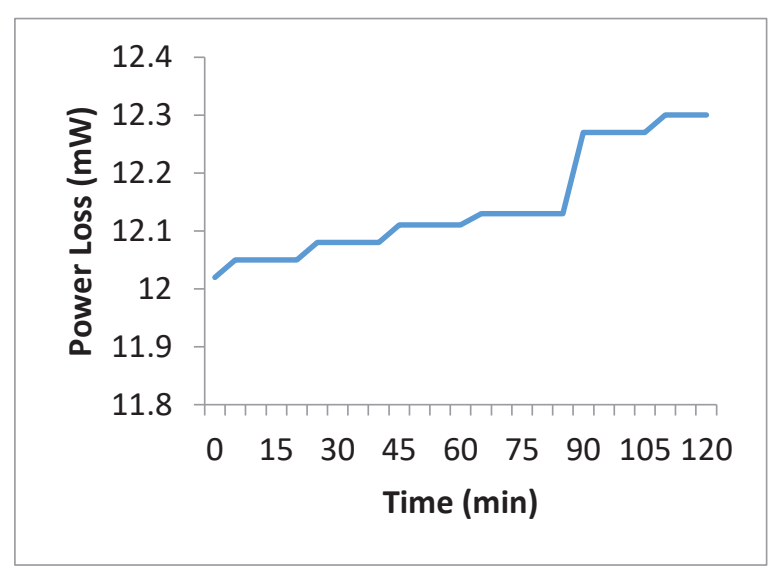

Fig. 3. Graph of power loss vs etching time of POF in $50 \%$ Acetone

A more concentrated acetone is more likely to dissolve or affect the surface fiber, which will result in tapered POF. Fig. 4 shows the macroscopic view of POF with $80 \%$ of acetone immersion, and the diameter of the fiber is observed to decrease to $990 \mu \mathrm{m}$. In Fig. 5, the immersion of $50 \%$ acetone gives a smoother surface compared to higher concentration of acetone. Moreover, the collision molecules of the acetone with the surface of POF become less reactive when the dilution of the acetone increases. This factor seems to result in a smooth and clear surface after it is lifted from the solution.

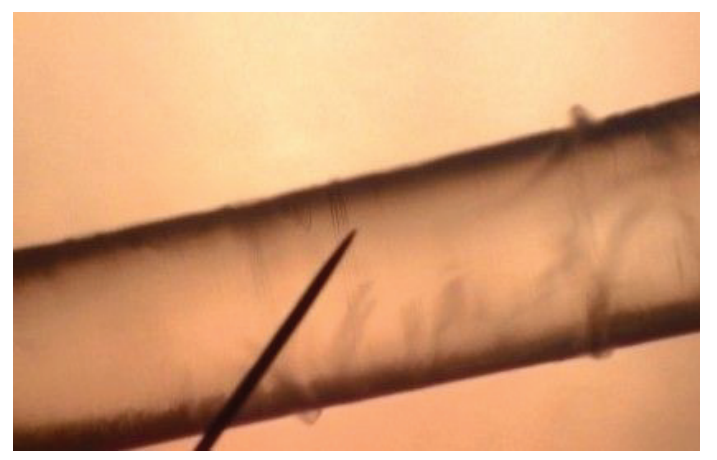

Fig. 4. Microscopic image of POF with immersion of $80 \%$ Acetone 


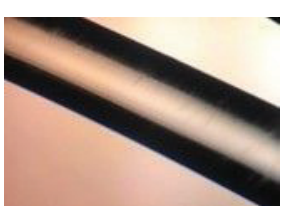

Fig. 5. Microscopic image of POF with immersion of $50 \%$ Acetone

\subsection{Effect of MIBK on Etching Rate}

MIBK is also an organic solvent that is very efficient in removing the cladding of polymer optical fiber such as acetone. Fig. 6 shows that around $210 \mathrm{~s}$ is needed to reach the fiber core when the solution for immersion is pure MIBK. Acetone appears to react faster compared to MIBK. These organic solvents can be used in the etching process of POF because it becomes susceptible to brittle stress fracture for a short while. In $80 \%$ concentration, the power loss increases after $3 \mathrm{~min}$ and it reaches the fibers core around $4.5 \mathrm{~min}$. Next, the power loss increases abruptly as it reaches and etches the core as shown in Fig. 7. For $50 \%$ of MIBK, the time taken to etch POF is longer as the concentration of the solution decreases. The power loss becomes much faster when the fiber is immersed in $80 \%$ compared to $50 \%$ of MIBK, in which it takes around $10.07 \mathrm{~min}$ to reach the fibers core as shown in Fig. 8.

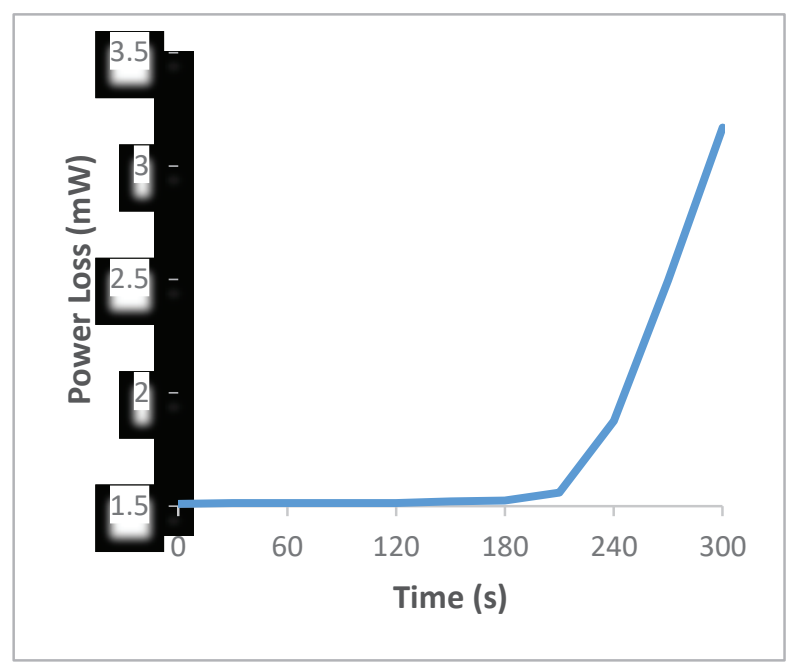

Fig. 6. Graph of power loss vs etching time of POF in $100 \%$ MIBK

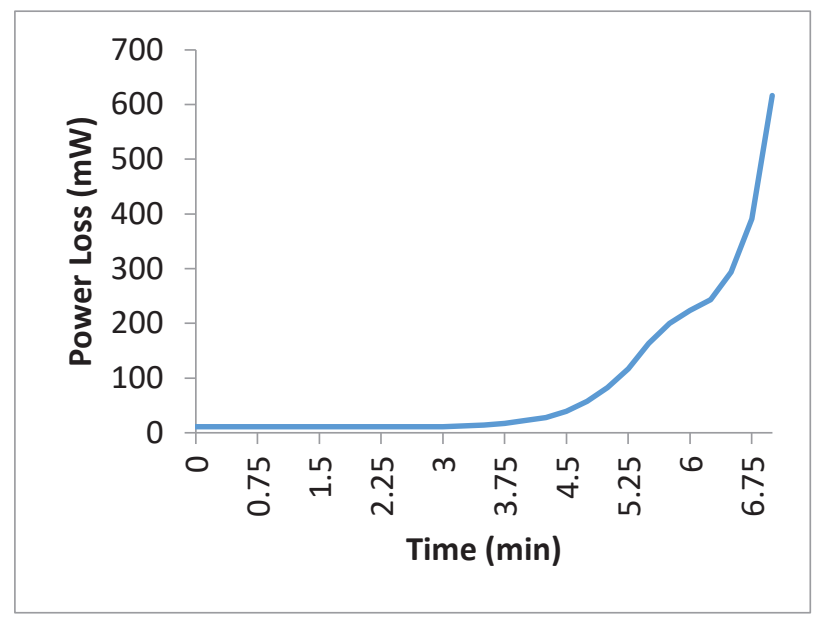

Fig. 7. Graph of power loss vs etching time of POF in $80 \%$ MIBK

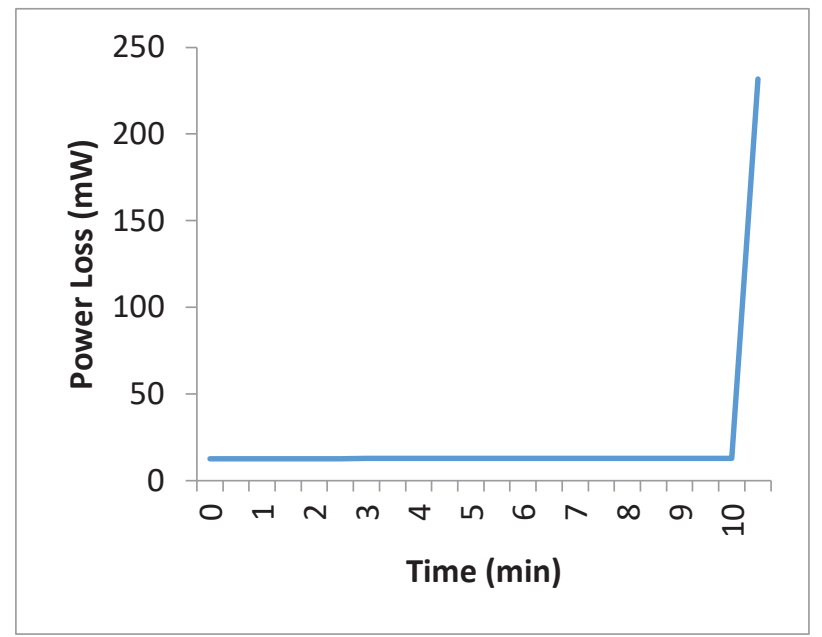

Fig. 8. Graph of power loss vs etching time of POF in 50\% MIBK

Fig. 9 shows the microscope image of unclad POF when it is immersed in $50 \%$ of MIBK solution. As can be seen, it has a rougher surface compared to when it is immersed in $50 \%$ of acetone. The surface roughness in the immersion of $50 \%$ and $80 \%$ of MIBK is quite similar. The POF respectively reduce the diameter to 970 $\mu \mathrm{m}$ and $980 \mu \mathrm{m}$ for $50 \%$ and $80 \%$ of MIBK.

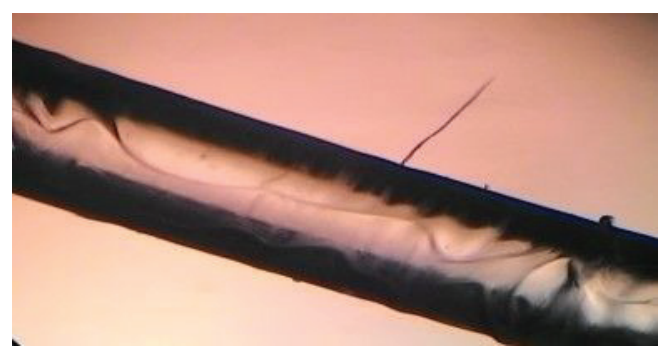

Fig. 9 Microscopic image of POF with immersion of $80 \%$ MIBK 


\subsection{The Effect of Acetone-Methanol Mixture on Etching Rate}

Fig. 10 shows POFs that are etched through the immersion in a mixture of acetone and methanol by ratio $(2: 1)$. The power output loss stops to increase slowly after 50 minutes of immersion, and no changes can be observed until the two hours of immersion ends at 503.5 $\mathrm{mW}$. Acetone plays an important role in etching due to its extremely reactive nature towards the polymer. For this particular solution, the mixture of the solution managed to produce tapered POF but with longer time compared to acetone and MIBK. Generally, acetone which is ketone can be mixed with any solvent including methanol. The function of methanol in the solution is to enhance the reactivity of the solvent towards the fiber surface.

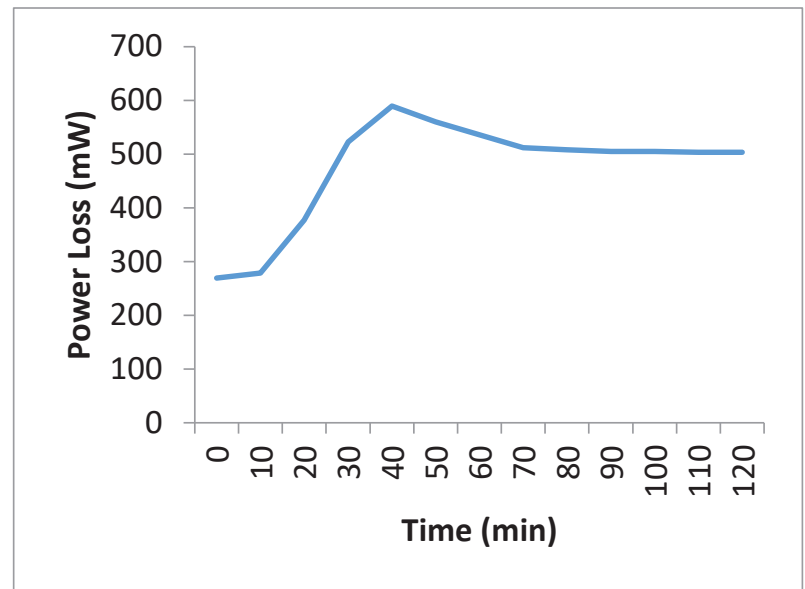

Fig. 10 Graph of power loss vs etching time of POF in the mixture of Acetone and Methanol (40:20)

For the surface structure of acetone-methanol mixture, the fiber fails to etch smoothly based on the presence of residue on the surface of POF after being immersed into the solution after 2 hours as shown in Fig. 11. This is in contrast with the microscopic image of acetone shown in Fig. 4, which produces smooth and clean surface after being immersed in $50 \%$ of acetone with the same 2 hours duration as applied in acetonemethanol mixture.

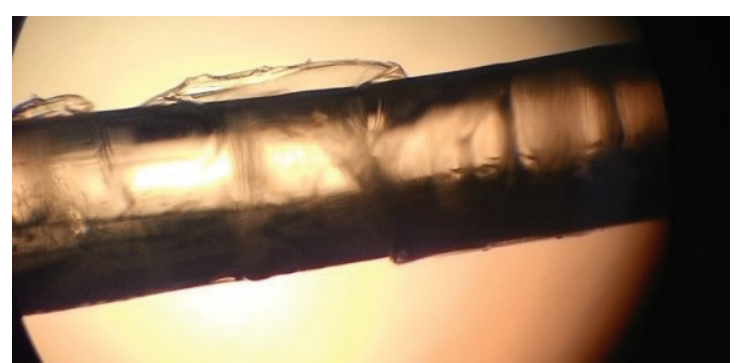

Fig. 11. Microscopic image of POF with immersion of mixture of acetone and methanol

\subsection{Measurements of Ethanol Solution on Unclad POF}

The initial loss of this sample is fixed at $11.16 \mathrm{dBm}$ when immersed in ethanol concentration. POF sample unclad with $100 \%$ of acetone solution is immersed in different concentrations of ethanol solution. Fig. 12 shows the graph of power output reading versus the concentration of ethanol, whereby higher concentration tends to produce lower power output. Moreover, the evanescent absorption coefficient increases when the concentration increases. The evanescent field penetrates into both solution and evanescent wave absorbance occurs when the unclad POF is immersed in ethanol solution, in which it will increase as the concentration of solution increases [8]. The refractive index of the ethanol also increases when the concentration percentage is increased [9]. According to Khijwania and Gupta [10], the increase of penetration depth can result in the increase of absorbance of evanescent field by the absorbing medium, which can lead to the increase of sensitivity of the sensor.

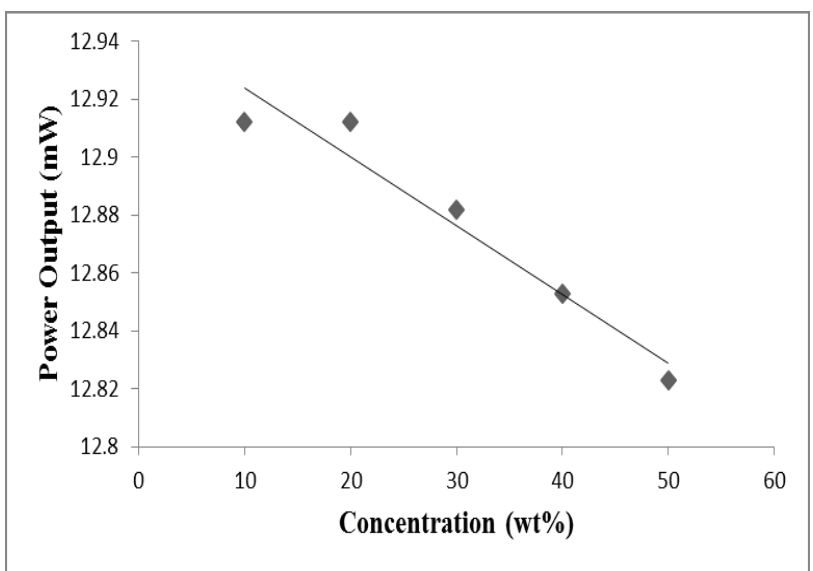

Fig. 12. Concentration of ethanol versus power output reading

\section{Conclusion}

In the evanescent wave fiber optic sensor, the cladding of the optical fiber is removed and made to be in direct contact with the evanescent wave. Moreover, any materials which are capable of absorbing the particular wavelength of the incoming light inside the optical fiber will take the energy out of the evanescent wave. Hence, the higher the concentration of the acetone and MIBK, the faster is the time taken for the fiber to break because the fiber becomes very brittle easily. Acetone which acts as the etching solution can give a smoother surface compared to MIBK and mixture acetone-methanol. Therefore, it is better for the etching solution to dilute with distilled water even though the etching rate will be longer. Finally, the surface quality will be good for sensing application because the rough surface which is formed on POF will affect the waveguiding process. 
This project was sponsored by the Kementerian Pendidikan Malaysia (KPM) through grant scheme (USIM/RAGS/FST/36/50215) and Universiti Sains Islam Malaysia (USIM) through grant schemes (PPP/USG0115/FST/30/12315).

\section{References}

1. Kara Peters, Smart Material and Structures, 20 013002, pp. 17 (2011)

2. Kuzyk, Mark G., Polymer Fiber Optics: Materials, Physics, and Applications (1st Ed) pp. 7-10 (Florida: CRC Press, 2007)

3. Batumalay, M; Lokman, A; Ahmad, F; Arof, H; Ahmad, H; Sulaiman, W.H., Sensor Journal, 13(12), pp 4702-4705 (2013)

4. D.F. Merchant, P.J. Scully, and N.F. Schmitt., Sensor and Actuators, 76: 365-371 (1999)

5. SM John, Evanescent wave fibre optic sensors: theory - Shodhganga (2011)

6. N. Punjabi, J. Satija, and S. Mukherji, Smart Sensors, Measurement and Instrumentation, pp. 2545 (2015)

7. X. Hu, C. F. J. Pun, H. Y. Tam et al., Optics Express. 22 (15) : 18807-18817 (2014)

8. P Suresh Kumar, CPG Vallabhan, VPN Nampoori, VN Sivasankara Pillai and Radhakrishnan, Journal of Optics A: Pure and Applied Optics, 4 pp. 247-250 (2002)

9. J. Nowakowska, The refractive indices of Ethyl Alcohol and Water Mixtures, Loyola University Chicago (1939)

10. S.K Khijwania, B. D. Gupta., Optic Communication, 152(4-6), pp. 259-262 (1998) 